\title{
Introduction to the Issue on Filtering and Segmentation with Mathematical Morphology
}

This issue presents novel contributions and introduces the state-of-art in filtering and segmentation methods using mathematical morphology. Historically, mathematical morphology developed a theoretical framework for non-linear image analysis, which from its inception led to important theoretical and practical results. Due to its algebraic foundation and geometrical intuition, the theory of mathematical morphology is very flexible, capable of handling many diverse image types, including (but not limited to) classical multi-dimensional signals as well as general graphs and surfaces.

We received 24 submissions from all over the world, from which we selected 13 articles for publication in this issue spanning a large segment of the research in morphological filtering and segmentation. The first article is an invited paper authored by Jean Serra, who is one of the two founders of Mathematical Morphology and a recent special conference in his honor has been the inspiration for this issue. In a "Tutorial on Connective Morphology", Jean Serra explores morphological operators from the perspective of connections: set connections, connective segmentations, connected operators, hierarchies of partitions and optimal cuts are the five key concepts under scrutiny.

The article "Random Projection Depth for Multivariate Mathematical Morphology," by S. Velasco-Forero and J. Angulo, considers a projection depth map for vector ordering, whereby the authors study a statistical depth function that approximates the Mahalanobis distance from a vector cloud, and use the function in applying mathematical morphological tools to multivalued (vector) images.

The article "Non-Local Morphological PDEs and p-Laplacian Equation on Graphs with Applications in Image Processing and Machine Learning," by A. Elmoataz et al., investigates connections between non-local morphological PDEs, p-Laplacian equation and non-local average filtering on graphs. It introduces a new class of non-local p-Laplacian operators, involving discrete morphological gradient on graphs, as solutions to several inverse problems in image processing.

The article "Active Contours on Graphs: Multiscale Morphology and Graphcuts," by K. Drakopoulos and P. Maragos, proposes two methods for computing geodesic active contours on arbitrary graphs, based on several ideas stemming from differential morphology: (i) approximations to the calculation of the gradient and the divergence of vector functions defined on graphs and uses these approximations to apply the technique of geodesic active contours for object detection on graphs; (ii) appropriate weights are calculated for each edge for which the Riemannian length of a contour can be approximated by the weighted sum of intersections of the contour with the edges of the graph and applied in a graphcut-based solution to the geodesic active contour problem on graphs.

The article "First Departure Algorithms and Image Decompositions into Peaks and Wells", by F. Meyer, presents viewpoint-dependent algorithms to reversibly decompose an image 
function into peaks and wells based on a generalized shortest path algorithm on weighted graphs. These algorithms include reconstruction closings or floodings and are illustrated with applications.

The article "Salience Adaptive Structuring Elements," by V. Curic et al., introduces salienceadaptive structuring elements that adjust both their shape and their size according to the salience of the edges of an image. Consequently, morphological operators using these kinds of structuring elements degrade edges with high salience to a lesser extent than those with low salience.

J.-C. Pinoli and J. Debayle authored the article "Spatially and Intensity Adaptive Morphology" in the context of General Adaptive Neighborhood Image Processing (GANIP). Structuring elements are spatially varying, and their activity is controlled according to the image intensities (level sets are processed at different scales). The advantages of these adaptive operators are highlighted on real-world application examples for image background removal, image restoration and image enhancement.

The article "Efficient Robust d-dimensional Path Operators," by F. Cokelaer et al., deals with morphological operators that use constrained paths as structuring elements. These paths-based operators are very efficient at filtering thin and/or oriented structures in images. Specifically, this article introduces a novel notion of path robustness, allowing the corresponding operators to better and more efficiently deal with noise, in 2D and 3D images. The authors illustrate applications of the proposed approach to blood vessel filtering and segmentation.

The article "One-Dimensional Openings, Granulometries and Component Trees in O(1) per Pixel," by V. Morard et al., provides a novel algorithm to compute one-dimensional openings, granulometries, and 1-D component trees. Its complexity is linear with respect to image dimension and constant with respect to the opening cycle.

The short article "Fast Morphological Image Processing Open-Source Extensions for GPU processing with CUDA," by M. Thurley and V. Danell ${ }_{2}$ provides a framework that allows 32bit processing of large-scale images to deliver open-source extensions for GPU enabled morphological image processing based on CUDA. The implementation of vHGW algorithms on a GPU to perform fast morphological operations on images by using analog processing is useful for analysis and processing of images with more than 8 bits.

The article "Classification of Remote Sensing Optical and LiDAR Data Using Extended Attribute Profiles," by M. Pedergnana et al., proposes a technique for feature extraction and supervised classification architecture used for classifying multisensor imagery composed of multi/hyperspectral channels and LIDAR data. This approach is novel to remote sensing and emphasizes the EAP-SVM-RF formulation: (i) to prevent Hughes' effects, the Extended Attribute Profile (EAP) approach was applied first to extract features from the multi/hyperspectral and LIDAR data, (ii) vector stacking is applied to the two sets of EAP features, and (iii) SVM and Random Forest (RF) are applied to classify in the stacked-vector space.

The article "Morphology-Based Crack Detection for Steel Slabs," by A. Landström and M. Thurley, is an application of mathematical morphology to continuous casting. Continuous casting is a highly efficient process used to produce most of the world steel production tonnage, but can crack in the semi-finished steel product. The authors present an approach for 
an automated on-line crack detection system, based on 3D profile data of steel slab surfaces, utilizing morphological image processing and statistical classification.

The article "Multiple Luminaire Identification in Airborne Images of Airport's Approach Lighting Using Mathematical Morphology With Variable Length Structuring Element," by S. Chowdhury et al., proposes analysis of the pattern of the approach-lighting system at an airport, used to provide identification and guidance to pilots from a distance. The proposed approach is based on morphological operators with a variable-length structuring element (VLSE). A novel technique for automatic determination of the VLSE is proposed and allows successful identification of the luminaires from the image data as verified through the use of simulated and real data.

As is apparent from the description above, the articles in this issue span a wide range of important topics using innovative methods based on morphological filters. We hope that the publication of this issue will promote further research and inspire new approaches to address interesting and important challenges using methods and techniques from mathematical morphology.

In closing, we would like to thank all the authors who submitted research manuscripts to this issue, as well as the reviewers for lending their time to evaluate manuscripts and providing valuable comments and suggestions. We would like to extend a special note of gratitude to Dr. Vikram Krishnamurthy, Editor-in-Chief of the Journal of Selected Topics in Signal Processing, and Ms. Rebecca Wollman, IEEE Publications Coordinator, for their constant support.

\author{
Laurent Najman (Lead Guest Editor) \\ Université Paris-Est, \\ Laboratoired'Informatique Gaspard Monge, \\ Equipe A3SI, ESIEE Paris, France \\ Email: l.najman@esiee.fr \\ URL: http://www.laurentnajman.org
}

\title{
Junior Barrera
}

Computer Science Department

Institute of Mathematics and Statistics, University of

São Paulo, 05508-090

São Paulo SP, Brazil

Email: j.barrera@uol.com.br

URL: http://www.vision.ime.usp.br/ jb/

\section{B. S. Daya Sagar}

Systems Science and Informatics Unit

Indian Statistical Institute

$8^{\text {th }}$ Mile, Mysore Rd, RVCE PO

Bangalore 560 059, India.

Email: bsdsagar@isibang.ac.in

URL: http://www.isibang.ac.in/ bsdsagar

\section{Petros Maragos}

School of Electrical and Computer Engineering

National Technical University of Athens

Athens, 15773, Greece

Email: maragos@cs.ntua.gr

URL: http://cvsp.cs.ntua.gr/maragos/

\section{Dan Schonfeld}

Department of Electrical \& Computer Engineering Department of Computer Science

Department of Bioengineering

University of Illinois at Chicago

Room 1020 SEO (M/C 154)

851 South Morgan Street

Chicago, IL 60607-7053

Email: dans@uic.edu

URL: http://www.ece.uic.edu/ ds 